\title{
Multiple Sclerosis of the Spinal Cord: is Gadolinium Irreplaceable in Assessing Lesion Activity?
}

\author{
Treabă Constantina Andrada1', Buruian M11, Bălașa Rodica², Podeanu Maria Daniela1', Simu IP1, Macavei ${ }^{2}$ \\ 1 Department of Radiology and Imaging, County Emergency Clinical Hospital, Faculty of Medicine, University of Medicine and Pharmacy, Tîrgu Mureș, Romania \\ 2 Department of Neurology, County Emergency Clinical Hospital, Faculty of Medicine, University of Medicine and Pharmacy, Tîrgu Mureș, Romania
}

\begin{abstract}
Purpose: To evaluate the relationship between the T2 patterns of spinal cord multiple sclerosis lesions and their contrast uptake.
Material and method: We retrospectively reviewed the appearance of spinal cord lesions in 29 patients (with relapsing-remitting multiple sclerosis) who had signs and symptoms of myelopathy on neurologic examination and at least one active lesion visualized on magnetic resonance examinations performed between 2004 and 2011. We correlated the T2 patterns of lesions with contrast enhancement and calculated sensitivity and specificity in predicting gadolinium enhancement.

Results: Only focal patterns consisting of a lesion's center homogenously brighter than its periphery on T2-weighed images (type I) correlated significantly with the presence of contrast enhancement $(p=0.004)$. Sensitivity was 0.307 and specificity 0.929 . In contrast, enhancement was not significantly related to uniformly hyperintense T2 focal lesions (type II) or diffuse (type III) pattern defined as poorly delineated areas of multiple small, confluent, subtle hyperintense T2 lesions ( $p>0.5$ for both).

Conclusions: We believe that information about the activity of multiple sclerosis spinal cord lesions in patients with myelopathy may be extracted not only from contrast enhanced, but also from non-enhanced magnetic resonance images.
\end{abstract}

Keywords: spinal cord, multiple sclerosis, magnetic resonance imaging

Received: 13 April 2012

\section{Introduction}

In as many as $90 \%$ of multiple sclerosis (MS) patients, the spinal cord is involved [1].

It is generally believed that since acute multiple sclerosis lesions are associated with transient breakdown of bloodbrain barrier (BBB), gadolinium contrast agents will produce enhancement of these lesions on T1 weighted images. Contrast enhancement remains a sensitive method for detection of active MS lesions with all the implications derived from this presence (diagnosis, clinical status and prognosis). But is the information offered by the administration of contrast really irreplaceable? Besides, it has been reported that contrast-enhancement might be influenced by the effect of drugs [2]. Therefore, the present study aims to identify imaging findings that predict lesion activity without contrast media by analyzing morphology and signal patterns of spinal cord MS lesions on T2-weighted images and correlating these with findings obtained from contrast-enhanced T1-weighted images.

\section{Material and method}

We retrospectively reviewed the appearance of spinal cord MS lesions in 29 patients ( 3 men and 26 women aged 2354 years) with relapsing-remitting MS, who had signs and symptoms of myelopathy on neurologic examination and at least one active lesion visualized on magnetic resonance (MR) examinations performed between January 2004 and

Correspondence to: Andrada Treabă

E-mail: andrada.treaba@umftgm.ro
December 2011. MR imaging was performed on $1.0 \mathrm{~T}$ (General Electric Medical Systems, Milwaukee, USA) using a phased-array multicoil. All patients underwent sagittal T2-weighted fast spin-echo images (TR/TE $=3000 / 102$ $\mathrm{ms})$ and sagittal T1-weighted spin-echo (TR/TE $=450 / 14$ $\mathrm{ms}$ ) images before and after the injection of $0.1 \mathrm{mmol} /$ $\mathrm{kg}$ gadopentetate dimeglumine. Postcontrast T1-weighted images were obtained through the spinal cord segments 5 to 10 minutes after the administration of contrast. For all images a 3-mm section thickness, a $256 \times 256$ matrix and an FOV of $24 \mathrm{~mm}$ were used.

\section{Data analysis}

To reduce interobserver variability all analyses were performed by two radiologists who worked together. Consensus was reached by agreement.

The T2 patterns of lesions were classified and divided into three types according to their morphology and signal intensity. When focal, lesions were judged according to their longest diameter.

Each lesion was then analyzed on pre- and postcontrast T1-weighted images to evaluate the presence of contrast uptake and its pattern. The questionable, small areas of enhancement near the pial surface were excluded from the analysis. Fisher's exact test was used to explore the association between the T2 type of lesion and contrast enhancement on postcontrast T1-weighted images. A p value less than 0.05 was considered statistically significant. Sensitivity and specificity in predicting gadolinium enhancement was also calculated. 

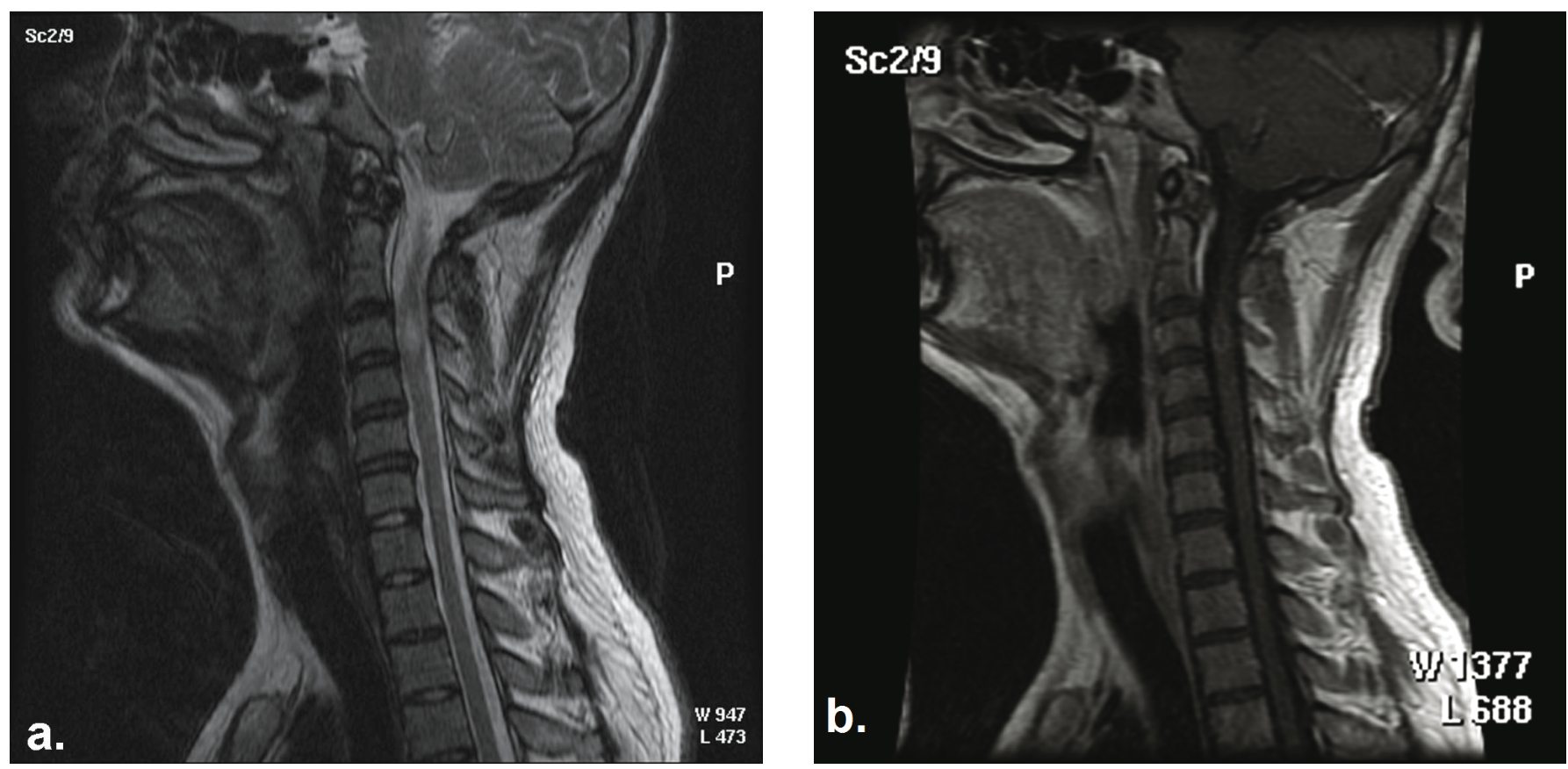

Fig. 1. Type I pattern spinal cord lesion on T2 weighted image (a) associated with ring-like enhancement on postcontrast T1-weighted image (b).

\section{Results}

A total of 96 MS lesions (90 focal and 6 diffuse) were identified. Thirty-nine (40.65\%) lesions showed contrast enhancement on postcontrast T1-weighted images (Table I).

The focal pattern consisting of a lesion's center homogenously brighter than its periphery on T2-weighed images (type I) correlated significantly $(\mathrm{p}=0.004)$ with the presence of contrast enhancement. Sensitivity was 0.307 and specificity 0.929 .

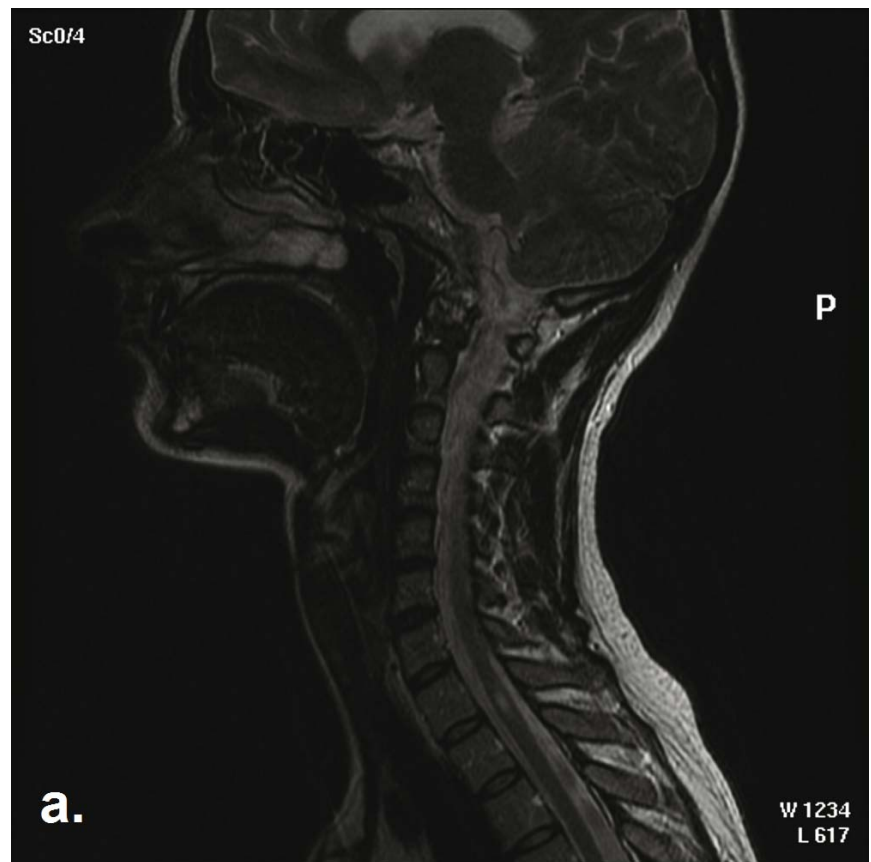

The corresponding contrast enhancement pattern was ring-like $(58.33 \%$ - predominant), nodular $(25 \%)$ or other (e.g. arc-like, $16.66 \%$ ). The ring-like enhancement was linked to lesions measuring more than $6 \mathrm{~mm}$. Typical examples are presented in Figures 1 and 2. Associated spinal cord enlargement (swelling) was observed in 5 lesions. There was no significant $(\mathrm{p}=0.145)$ correlation between the uniformly hyperintense focal lesions on T2-weighted images (type II) and contrast load (Figure 3). Enhancement when appeared associated with this pattern (Figure

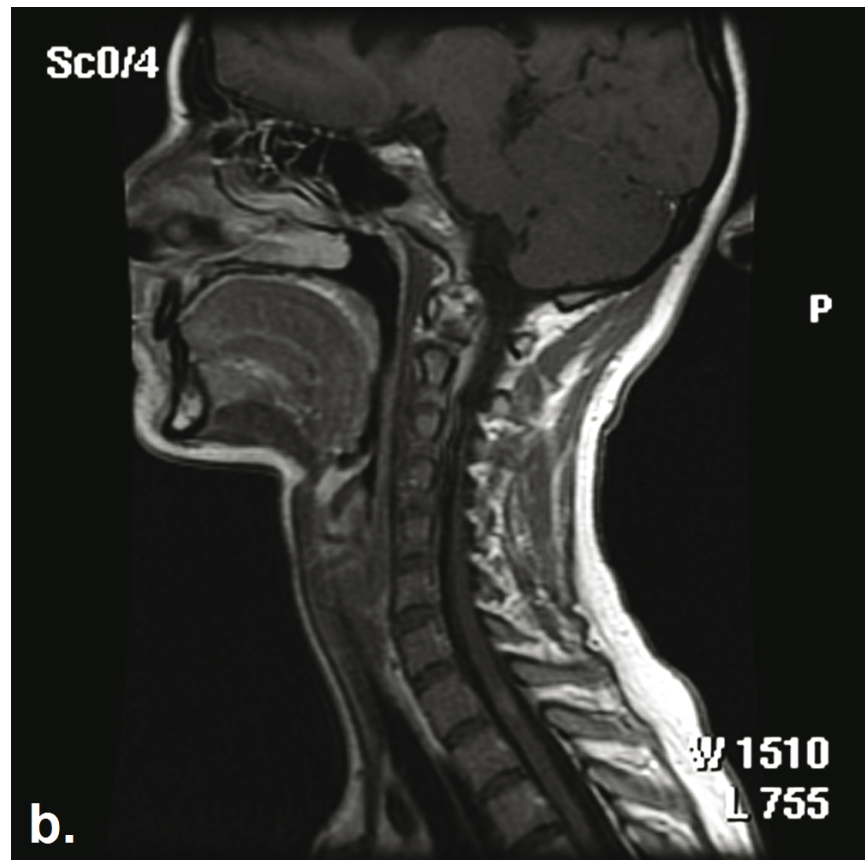

Fig. 2. Type I pattern spinal cord lesion on precontrast T2-weighted image (a) shows homogeneous nodular enhancement on postcontrast T1-weighted image (b). 

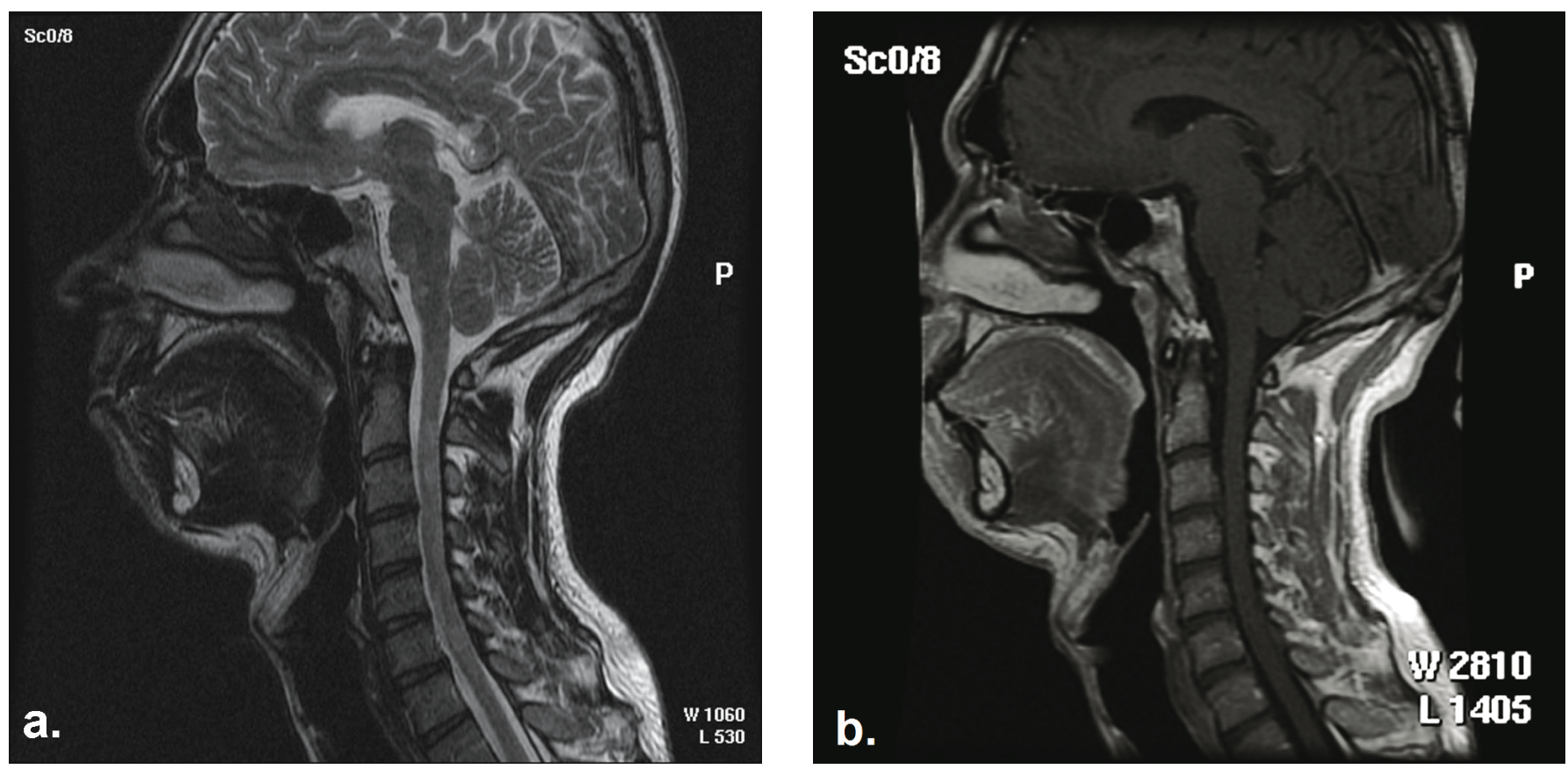

Fig. 3. Multiple, type II pattern, spinal cord lesions are seen on T2-weighted image (a). Contrast enhancement is not observed on gadolinium enhanced T1-weighted image (b).

4) was mostly patchy (85.18\%). Enhancement was not significantly $(\mathrm{p}=0.078)$ related to diffuse pattern (type III) defined as areas with poorly delineated, confluent, multiple small, subtle hyperintense T2 lesions scattered throughout the cord (Figure 4).

\section{Discussions}

Previous studies showed that contrast enhancement is associated with the presence of an active inflammatory process with perivascular lymphocytic infiltration [3].

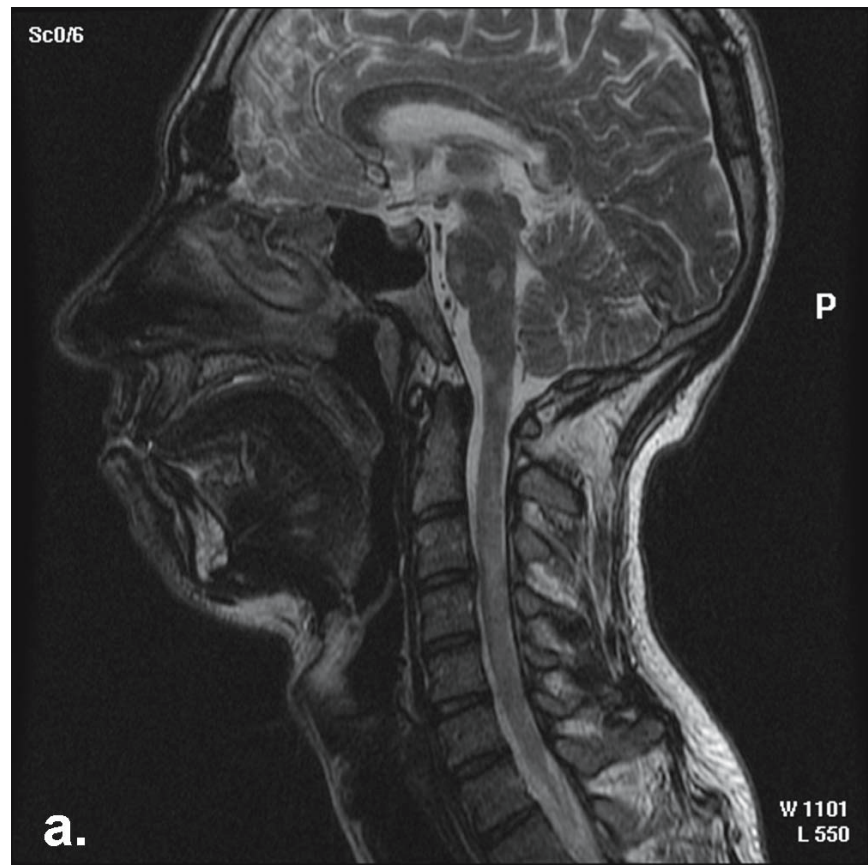

We found that the T2 pattern of spinal cord lesion which correlated best with contrast enhancement was the one consisting of a focal lesion with its center homogenously brighter than its periphery. This less T2 hyperintense periphery, that extended beyond both the lesion's center and the borders of the enhancing component of the lesion, most likely represents reversible, perilesional edema. The presence of peripheral edema may also explain the associated spinal cord enlargement encountered in some of our cases.

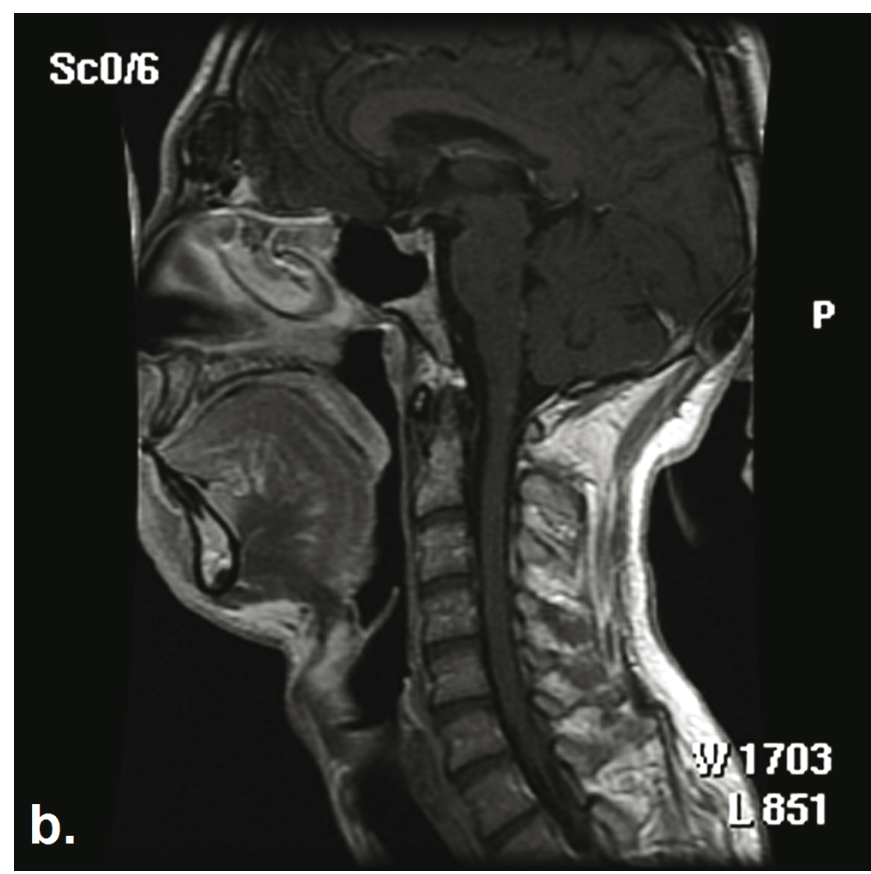

Fig. 4. Multiple lesions are present on T2-weighted image (a) within the cervical spinal cord corresponding to pattern type II (at foramen magnum level) and III (extending from C3 to C5). The posteriorly located type II lesion shows patchy enhancement on postcontrast T1weighted image but enhancement is not observed in the pattern type III lesion (b). 
Ring-like enhancement probably arises from recent inflammation at the edge of chronic demyelinating lesions [2].

However, not all spinal cord lesions presenting this T2 pattern enhanced in our patients. It is well known that in the acute inflammatory phase, the lesion disrupts the $\mathrm{BBB}$, leading to gadolinium enhancement. Although this is considered to be one of the earliest changes observed in MS lesions, neuropathological and immunocytochemical studies revealed that BBB leakage can be found to variable degrees in every MS lesion [4]. Quantitative contrastenhanced MRI also showed that subtle BBB leakage was a consistent feature in non-enhancing lesions [5]. Those observations suggest that enhancement cannot detect all inflammatory changes, particularly when the level of inflammation is low. Certainly, much activity is taking place that is not necessarily defined by enhancement alone [6].

Besides, contrast enhancement depends on many factors, including both dosing of contrast material and the time from injection to imaging. Late phase imaging and triple dosing may improve the conspicuity of acute lesions [7]. On the other hand, contrast administration cannot help to document disease activity following steroid or Binterferon treatment, when variable degree of suppression of enhancing lesions occurs [2]. There are also conditions when the administration of gadolinium-based agents is prohibited [8].

We found a weak correlation between the uniformly hyperintense focal lesions on T2-weighted images and contrast enhancement. The patchy enhancement, when encountered, may be a consequence of the transitory activity of MS lesions. If a lesion is a consequence of MS, a follow-up examination is expected to show disappearance or decrease in enhancement with time [9].

The diffuse pattern of MS spinal lesions was not correlated with contrast enhancement. However, MR imaging-histopathological correlations have shown that diffuse hyperintensities in the spinal cord correspond primarily to demyelination [3].

\section{Conclusions}

We believe that information about the activity of multiple sclerosis spinal cord lesions in patients with myelopathy may be extracted not only from contrast enhanced, but also from non-enhanced MR images.

The lesion's pattern consisted of a lesion's center homogenously brighter than its periphery on T2-weighed images correlated best with contrast enhancement and subsequent lesions activity.

\section{Acknowledgement}

This paper is partly supported by Sectorial Operational Programme Human Resources Development (SOP HRD), financed from the European Social Fund and by the Romanian Government under the contract number POSDRU 80641.

\section{References}

1. Bot JC, Barkhof F, Lycklama a Nijeholt G, et al. Differentiation of multiple sclerosis from other inflammatory disorders and cerebrovascular disease: Value of spinal MR imaging. Radiology. 2002;223:46-56.

2. Sahraian MA, Radue EW-MRI. Gadolinium enhancing lesions in multiple sclerosis, in Atlas of MS lesions. Springer-Verlag, Berlin, 2008, 45-65.

3. Nesbit GM, Forbes GS, Scheithauer BW, Okazaki H, Rodriguez M. Multiple sclerosis: histopathologic and MR and/or CT correlation in 37 cases at biopsy and three cases at autopsy. Radiology. 1991;180(2):467-474.

4. Lucchinetti CF, Brück WB, Rodriguez M, Lassmann $H$. Distinct patterns of multiple sclerosis pathology indicates heterogeneity in pathogenesis. Brain Pathology. 1996;6(3):259-274.

5. Soon D, Tozer DJ, Altmann DR, Tofts PS, Miller DH. Quantification of subtle blood-brain barrier disruption in non-enhancing lesions in multiple sclerosis: a study of disease and lesion subtypes. Multiple Sclerosis.2007;13(7):884-894.

6. He J, Grossman RI, Ge Y, Mannon LJ - Enhancing patterns in multiple sclerosis: evolution and persistence. AJNR Am J Neuroradiol. 2001;22: 664-669.

7. Yousry I, Filippi M, Walther E, Gasperini C, Capra R, Yousry, TA. Serial gadolinium-DTPA of spinal cord MRI in multiple sclerosis: triple vs. single dose. Magn Reson Imaging. 2000;18(9):1183-1186.

8. Dillman JR, Ellis JH, Cohan RH, Strouse PJ, Jan SC. Frequency and severity of acute allergic-like reactions to Gadolinium-containing iv contrast media in children and adults. Americal Journal of Roengenology. 2007;189(6):1533-1538.

9. Trop I, Bourgouin PM, Lapierre Y, et al. Multiple sclerosis of the spinal cord: diagnosis and follow-up with contrast-enhanced MR and correlation with clinical activity. AJNR Am J Neuroradiol. 1998;19:1025-1033. 